\title{
Vibration switch compensation for maglev flywheel battery of EV based on standard LMS algorithm
}

\author{
Hui Gao* and Xianhai Meng \\ Nanjing University of Posts and Telecommunications, Nanjing 210023, China \\ *Corresponding Author: gaohui2005@163.com
}

\begin{abstract}
In maglev flywheel system, unbalance interference signals synchronous with the flywheel frequency would be produced because the flywheel rotor quality is uneven. The signal can reduce system control precision and stability and make the system produce unbalance vibration, so the signal should be filtered to reduce the effects to the control system. A kind of vibration switching compensation control strategy is proposed based on the standard LMS algorithm and one PID controller. The flywheel real-time rotation frequency is looked at as the switching reference; different LMS algorithm step size and different PID controller parameters are adopted in different rotational frequency. Simulation and experiment results verify the feasibility and effectiveness of the switch compensation strategy, can reduce the control current amplitude, and make the flywheel rotate around the inertial principal axis as much as possible.
\end{abstract}

Keywords: maglev flywheel; vibration compensation; switch control; displacement sensor; standard LMS algorithm.

\section{INTRODUCTION}

Maglev flywheel is suspended by active magnetic bearings because they have some advantages over conventional bearings such as contact-free support, no mechanical friction, requiring no lubrication, long serving life, and operating at higher speeds (Xu Y. P. et al., 2016; Niemann, A. C. et al., 2013). Maglev flywheel has some other advantages as high specific energy, high power, fast charge and discharge, long service life, no any waste gas pollution, and environment friendly (Gao H. et al., 2015; Chen, S. C. et al., 2014). It has been applied to the fields in electric vehicles, electric power system, and aerospace (Raymond M. S. et al., 2015). In electric vehicles, the maglev flywheel can be as the battery solely to drive the car (Liu W. et al., 2016).

In maglev flywheel system, unbalance interference signals synchronous with the flywheel frequency would be produced because the flywheel rotor quality is uneven, which will originate unbalance vibration response and affect the storage energy of the flywheel battery and restrict its application in electric vehicles and other arenas (Fang, J. C. et al., 2015). So, the signal should be filtered to reduce the effects to the control system. As a kind of adaptive filter, Least Mean Square (LMS) algorithm has been used in the inertia force minimum compensation for active magnetic bearings system successfully (Yao, D. et al., 2015; Shi, J. et al., 2002). The maximum steady-state error can be decreased to about $85 \%$ in (Yao, D. et al., 2015), but (Shi, J. et al., 2002) only realized constant speed compensation because of the standard LMS algorithm can filter fixed frequency signal.

In this paper, through analyzing the filtering characteristic of standard LMS algorithm, a kind of vibration switching compensation control strategy is proposed based on the LMS algorithm and one PID controller, and the strategy is closed-loop feedback control. The control principle is the flywheel real-time rotation frequency looked at as the switching reference, and different LMS algorithm step size and different PID controller parameters are adopted in different rotational frequency. Simulation and experiment results verify the feasibility and effectiveness of the switch compensation strategy, can reduce the control current amplitude, and make the flywheel rotate around the inertial principal axis as much as possible. 


\section{APPLICATION ANALYSIS FOR STANDARD LMS TO MAGLEV FLYWHEEL COMPENSATION}

The standard LMS algorithm was first proposed by Widrow in 1975 year (Widrow , B. et al., 1975), as one kind of simple and small calculation adaptive filter algorithm; the algorithm has been widely used in many fields, such as adaptive network control (Bogdanovic, N. et al., 2014), radar (Ting, L. K. et al., 2004), system identification (Aliyu, M. et al., 2015), and signal processing (He L. et al., 2015).

\section{Standard LMS algorithm principle}

The principle of LMS algorithm is using gradient random descent method to achieve the objective function minimization, and guaranteeing the output of the mean square error on the surface of the performance decline (Feuer, A. et al., 1985; Martinek, R et al., 2017). Figure 1 gives the philosophy and architecture for the standard LMS algorithm.

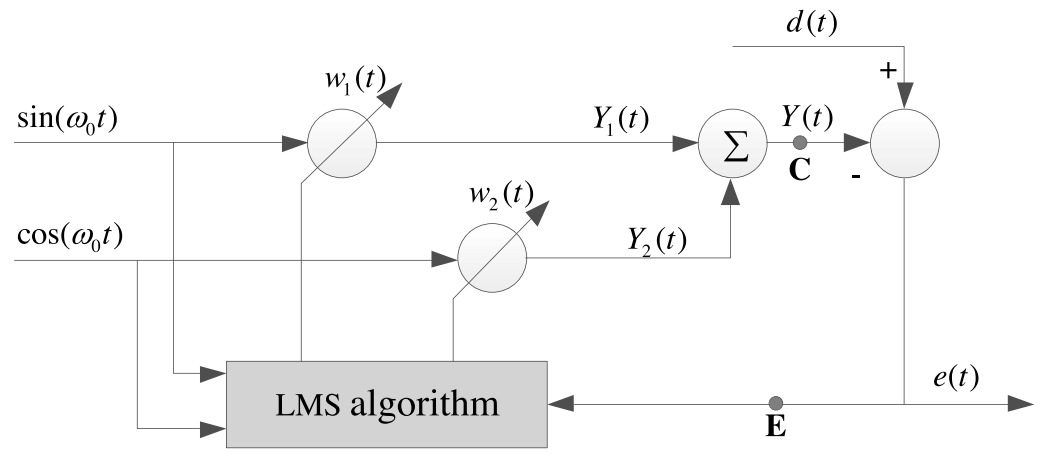

Figure 1. The standard LMS algorithm philosophy and architecture.

In Figure 1, $X(t)=\left[\sin \left(\omega_{0} t\right) \cos \left(\omega_{0} t\right)\right]$ is the input signal vector, $W(t)=\left[w_{1}(t) w_{2}(t)\right]^{T}$ is the weight vector, $Y(t)$ is learning signal, $Y_{1}(t)$ and $Y_{2}(t)$ are two components of $Y(t), d(t)$ is the desired signal, $e(t)$ is the error output signal, and $\omega_{0}$ is filter angle frequency.

The objective function of LMS algorithm is defined (Ghogho, M. et al,1998)

$\delta(t)=E\left[e^{2}(t)\right]$

where

$e(t)=d(t)-Y(t)=d(t)-X(t) W(t)$

$Y(t)=y_{1}(t) w_{1}(t)+y_{2}(t) w_{2}(t)$

Substituting Eq. (2) into Eq. (1), we obtain Eq. (3) as follows.

$\delta(t)=E\left[d^{2}(t)\right]+W(t)^{T} R W(t)-2 H^{T} W(t)$

where

$R=E\left[X(t) X^{T}(t)\right]$

$H=E[X(t) d(t)]$ 
$R$ and $H$ are the autocorrelation matrix and cross-correlation matrix, respectively. The gradient function of the objective function gradient can be written according to Eq. (4).

$\nabla \delta(t)=\frac{\partial \delta(t)}{\partial W(t)}=-2 H+2 R W(t)$

In Eq. (7), to reduce the calculation, the objective function gradient is replaced by the estimate mean square error gradient $\nabla e^{2}(t)$

$$
\nabla e^{2}(t)=\frac{\partial e^{2}(t)}{\partial W(t)}=-2 e(t) X(t)
$$

The weight vector iteration formula can be updated by Eq. (8)

$W_{k+1}(t)=W_{k}(t)-\frac{\mu}{2} \nabla e^{2}(t)=W(t)+\mu e(t) X(t)$

where $\mu$ is the fixed step size, and the following condition should be satisfied (Aliyu, M. et al., 2015):

$0<\mu<\frac{1}{\lambda_{\max }}$

where $\lambda_{\max }$ is the maximum eigenvalue; besides other constraints of the fixed step size can be calculated using the wave theory (Ghogho, M. et al., 1998)

$$
0<\mu<=\frac{2}{L \cdot \max \{s(\omega)\}}
$$

where $\mathrm{L}$ is the filter length, and $s(\omega)$ is the power spectrum peaks of the input signal.

If the following condition is satisfied, the LMS algorithm can implement adaptive filter for the desired signal:

$$
e(t)=\lim _{k \rightarrow+\infty}[d(t)-Y(t)] \rightarrow 0
$$

\section{Standard LMS algorithm adaptive filter analysis for maglev flywheel vibration compensation}

In view of the sine wave caused for the flywheel by the unbalance, the displacement sensor output signal is assumed as

$x_{s a}(t)=A(t) \sin \left(\omega_{r} t+\phi\right)=A(t) \sin \left(2 \pi f_{r} t+\phi\right)$

where $A(t)$ is the signal amplitude, $\omega_{r}$ is the flywheel angular frequency, $f_{r}$ is the flywheel rotation frequency, and $\phi$ is the initial phase determined by the flywheel initial position. Eq. (13) can be unfolded

$x_{s a}(t)=A(t) \sin \phi \cos \left(2 \pi f_{r} t\right)+A(t) \cos \phi \sin \left(2 \pi f_{r} t\right)$

Assuming $A(t) \sin \phi=a_{1}(t)$ and $A(t) \cos \phi=a_{2}(t)$, Eq. (14) can be rewritten

$x_{s a}(t)=a_{1}(t) \cos \left(2 \pi f_{r} t\right)+a_{2}(t) \sin \left(2 \pi f_{r} t\right)$

Here, the flywheel sine displacement signal $x_{s a}(t)$ is supposed to be the desired signal $d(t)$ and needs to meet Eq. (12) convergence. The single-channel signal discrete transmission is shown as Figure 2. 


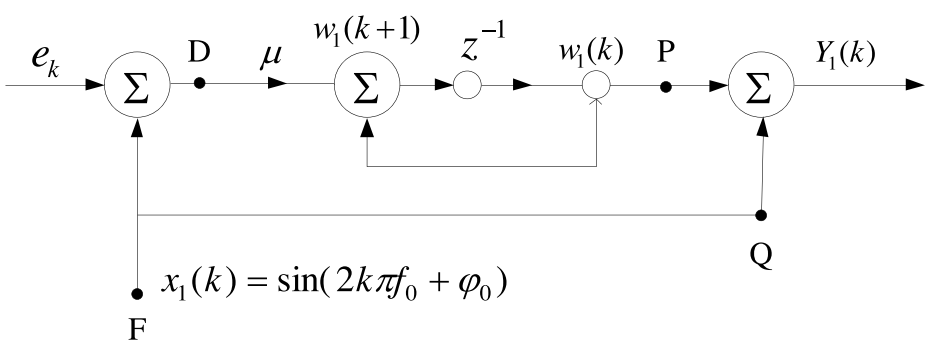

Figure 2. The single-channel signal transmission.

To analyze the isolation impulse response in Figure 2, assume there exits discrete unit impulse response if $k$ equals $n$, and the output function for $\mathrm{D}$ is

$$
h_{1}= \begin{cases}\sin \left(2 \pi n f_{0}+\varphi_{0}\right) & k=n \\ 0 & k \neq n\end{cases}
$$

The transfer function from $\mathrm{D}$ to $\mathrm{P}$ is $\mu /(z-1)$, and the impulse response is $h_{2}=\mu \cdot 1(k-1)$, where $1(k-1)$ is discrete unit step function. The output function of $\mathrm{P}$ is

$w_{1}(k)=h_{1} \otimes h_{2}=\mu \sin \left(2 \pi n f_{0}+\varphi_{0}\right)$

where $\otimes$ represents convolution, and the output function of $Y_{1}(k)$ is

$Y_{1}(k)=\mu \sin \left(2 \pi k f_{0}+\varphi_{0}\right) \sin \left(2 \pi n f_{0}+\varphi_{0}\right)$

The weight coefficients iterative function in Figure 2 are

$w_{1}(k+1)=w_{1}(k)+\mu e_{k} x_{1}(k)$

Similarly, the output function of $Y_{2}(k)$ in Figure 1 can be calculated

$Y_{2}(k)=\mu \cos \left(2 \pi k f_{0}+\varphi_{0}\right) \cos \left(2 \pi n f_{0}+\varphi_{0}\right)$

The discrete output function of $Y(t)$ in Figure 1 can be written according to Eq. (18) and Eq. (20)

$Y_{k}=Y_{1}(k)+Y_{2}(k)=\mu \cos \left[2 \pi(k-n) f_{0}\right]$

When the impact moment is 0 , the open loop unit impulse response from $\mathrm{E}$ to $\mathrm{C}$ in Figure 1 can be calculated

$Y_{k}=\mu 1(k-1) \cos \left(2 \pi k f_{0}\right)$

The z-transform of Eq. (22) is

$G_{L}(z)=\mu\left[\frac{z\left(z-\cos \left(2 \pi f_{0} T_{s}\right)\right)}{z^{2}-2 z \cos \left(2 \pi f_{0} T_{s}\right)+1}-1\right]=\mu \frac{z \cos \left(2 \pi f_{0} T_{s}\right)-1}{z^{2}-2 z \cos \left(2 \pi f_{0} T_{s}\right)+1}$

The close-loop transfer function from $\mathrm{d}(\mathrm{t})$ to $\mathrm{e}(\mathrm{t})$ can be calculated

$H_{L}(z)=\frac{E(z)}{D(z)}=\frac{1}{1+G_{L}(z)}=\frac{z^{2}-2 z \cos \left(2 \pi f_{0} T_{s}\right)+1}{z^{2}-2(1-\mu / 2) \cos \left(2 \pi f_{0} T_{s}\right)+(1-\mu)}$

where $T_{\mathrm{s}}$ is the sampling period, and the zero-point of Eq. (24) is $z_{0}=e^{ \pm 2 j \pi f_{0}}$. If the unbalance displacement frequency $f$ of the flywheel equals to the LMS algorithm input signal frequency $f_{0}$, the unbalance signal can be filtered by the algorithm, and Eq. (12) can be satisfied. 
To analyze the stability of the LMS close-loop system, the pole-point of Eq. (24) is given

$$
z_{p}=(1-\mu / 2) \cos \left(2 \pi f_{0}\right) \pm j\left[(1-\mu)-(1-\mu / 2)^{2} \cos ^{2}\left(2 \pi f_{0}\right)\right]^{1 / 2}
$$

The distance between the pole-point and the unit circle center is $(1-\mu)^{1 / 2}$, and the value is always less than 1 , so the close-loop always remained stable.

Here, assuming the desired signal is unit sinusoidal signal having $400 \mathrm{~Hz}$ frequency, $f_{0}$ also is $400 \mathrm{~Hz}, T_{s}$ is $5 \times 10^{-5} \mathrm{~s}$, and $\mu$ is 0.04 . The filter simulation using MATLAB is shown in Figure 3, where $d(k)$ is the desired signal, $Y(k)$ is the learning signal, and $e(k)$ is the error signal.

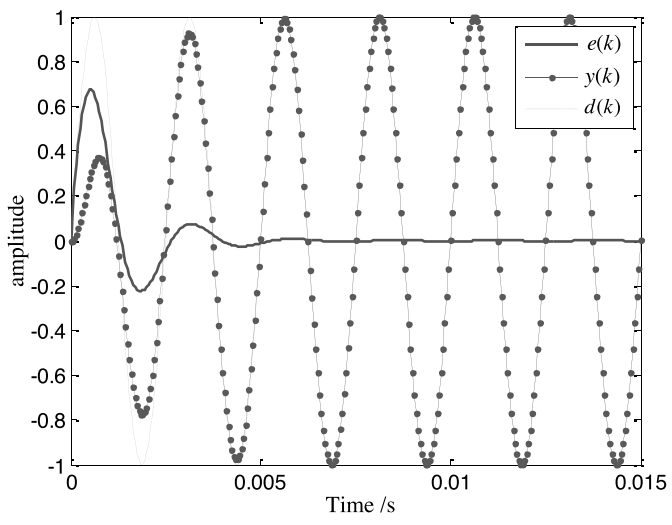

Figure 3. LMS algorithm filter simulation analysis.

The error signal amplitude reduced to about $25 \%$ of the desired signal after a period in Figure 3, to zero after about three periods, and satisfied convergence request. Because the LMS algorithm has iterative learning advantage, it can be used in the digital controller of maglev flywheel system, to achieve filter for the unbalance interference signals synchronous with the flywheel frequency.

In the application of vibration compensation for maglev flywheel system, the input signal frequency $f_{0}$ is fixed, but the flywheel rotational frequency $f$ is changing, so it exits frequency difference. The mean square error (MSE) function is given in Eq. (26) (Xiao, Y. et al., 1995).

$$
\operatorname{MSE}=E\left[e^{2}(t)\right]=\frac{A}{2}\left|H_{L}\left(e^{j 2 \pi f}\right)\right|^{2}=\frac{A}{2} \cdot \frac{4\left[\cos (2 \pi f)-\cos \left(2 \pi f_{0}\right)\right]^{2}}{(2-\mu)^{2}\left[\cos (2 \pi f)-\cos \left(2 \pi f_{0}\right)\right]^{2}+u^{2} \sin ^{2}(2 \pi f)}
$$

In Eq. (26), if $f_{0}$ equals $f$, MSE is zero; otherwise MSE is non-zero. Figure 4 gives the simulation analysis for MSE between different frequencies, assuming $f_{0}=400 \mathrm{~Hz}$, and $f=400 \mathrm{~Hz}$ or $380 \mathrm{~Hz}$.

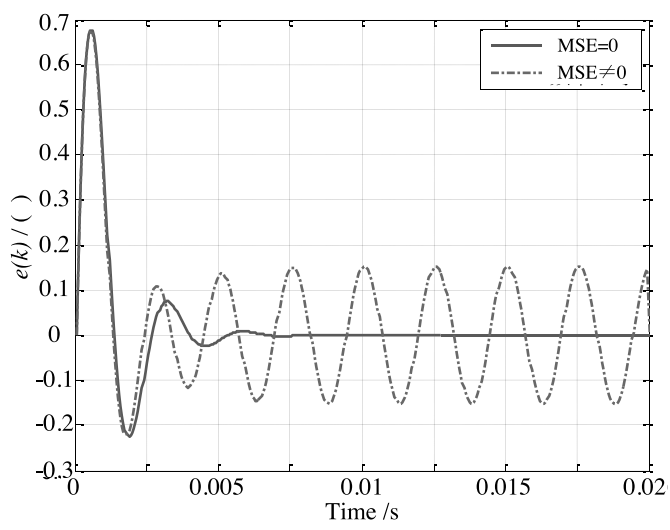

Figure 4. The simulation analysis for MSE between different frequencies. 
In Figure 4, if MSE is zero $\left(f_{0}=f\right)$, LMS algorithm error signal $e(t)$ ultimately closes to zero; it shows that it can implement effective filter. If MSE is not-zero $\left(f_{0} \neq f\right), e(t)$ exits oscillation, and the filter effect is halfway. The simulation result shows that the standard LMS algorithm only implements effective filter to fixed sine signals.

Besides, the influence of filtering effect about the step size $\mu$ to the standard LMS algorithm is also analyzed. Here, $\mu$ is assumed as $0.08,0.04$, and $0.004, f_{0}=f=400 \mathrm{~Hz}$, and Figure 5 shows the filtering effect.

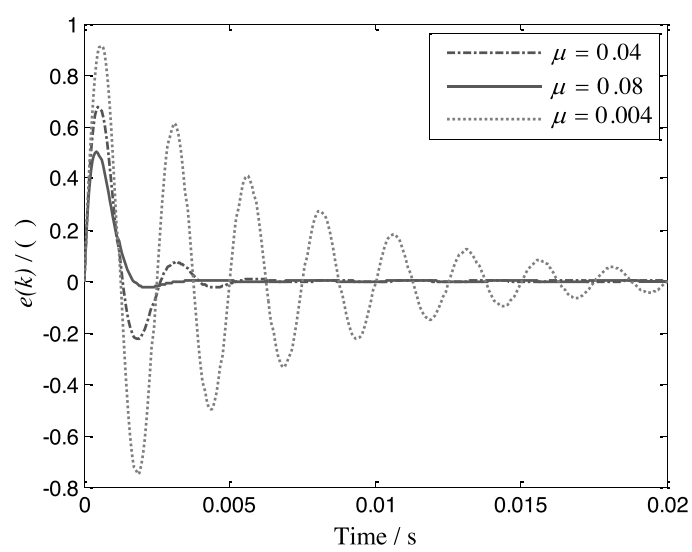

Figure 5. Filter effect simulation when $\mu$ selects different values.

If the step size $\mu$ is too small (0.004), the error signal has slower convergence speed, and the unbalance displacement signal cannot be filtered rapidly in the application of maglev flywheel vibration compensation. If $\mu$ is too big (0.08), the error signal has faster convergence speed, and it would make the maglev flywheel control system instable because the error signal cannot track the control signal well, and the phenomenon has been verified in maglev flywheel actual control test. Only when $\mu(0.04)$ is chosen relatively suitable for one fixed filter frequency, the error signal has faster convergence speed and better ability to follow the desired signal well.

\section{SWITCH COMPENSATION CONTROL USING STANDARD LMS ALGORITHM}

For the negative impact of the fixed step size $\mu$ in the vibration compensation application for flywheel unbalance, a kind of switch compensation control strategy is presented based on the standard LMS algorithm and one incomplete differential PID controller. In this strategy, some suitable $\mu$ are chosen according to the flywheel different rotating frequency, and corresponding groups PID parameters are selected; finally switch compensation control is executed. Figure 6 gives the switch compensation control diagram.

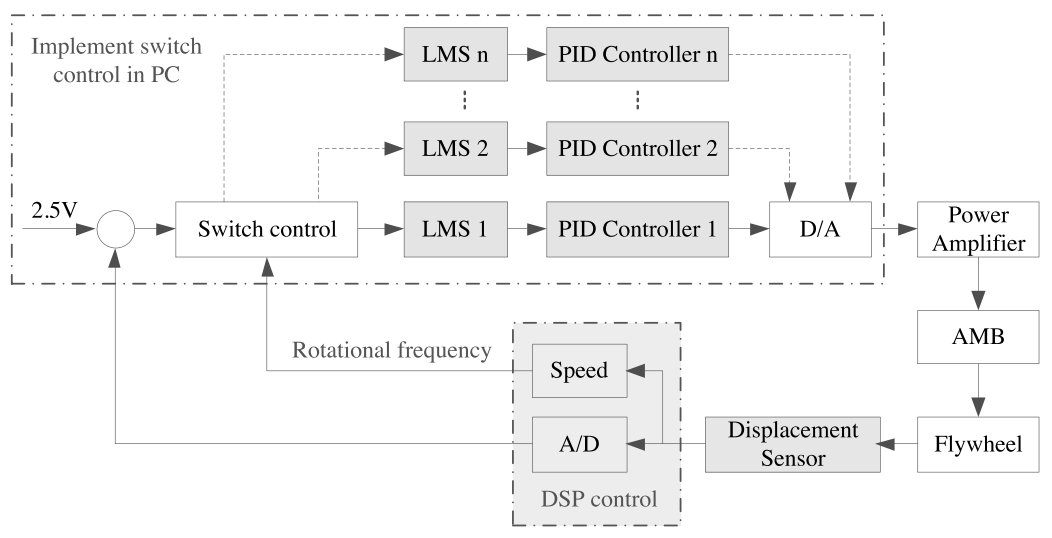

Figure 6. Switch compensation control using standard LMS algorithm for different parameters. 
In Figure 6, the standard LMS algorithm and the PID controller constitute some new filter controllers, named $C_{N}$, where the speed measuring unit and A/D transformation module are achieved by DSP chip, and the switch compensation algorithm is implemented PC. The PID controller transfer function is

$$
G_{P I D}(s)=K_{p}\left(1+\frac{1}{T_{i} s}+\frac{T_{d} s}{1+T_{f} s}\right) \cdot \frac{T_{l} s+1}{\alpha T_{l} s+1}
$$

where $K_{p}$ and $T_{i}$ are the scaling and integral coefficients, respectively, $T_{d}$ is the time coefficient, $T_{d s} /\left(1+T_{f s}\right)$ is the incomplete differential part, and $\left(T_{l}+1\right) /\left(\alpha T_{l s}+1\right)$ is the advanced link.

According to Eq. (24) and Eq. (27), the open-loop transfer function of $C_{N}$ is

$$
\begin{aligned}
& C_{N B}(z)=\frac{1}{1-H_{L}(z) \cdot G_{P I D}(z)} \\
& \text { where } G_{P I D}(z)=\left.G_{P I D}(s)\right|_{z=\frac{1+s}{1-s}} .
\end{aligned}
$$

\section{SIMULATION ANALYSIS}

The filtering effect of $C_{N}$ is analyzed using MATLAB. The PID parameters are selected in Table 1, and $T_{S}=0.00005 \mathrm{~s}, \mu=0.04, f_{0}=400 \mathrm{~Hz}$. The close loop transfer function bode diagram is established in Figure 7 for different PID parameters in A and B.

Table 1. PID controller parameters.

\begin{tabular}{ccccccc}
\hline parameters & $K p$ & $T_{i}$ & $i_{d}$ & $T_{f}$ & $T_{l}$ & $\alpha$ \\
$\mathrm{A}$ & 0.855 & 0.545 & 0.569 & 0.7539 & 0.0437 & 0.00537 \\
$\mathrm{~B}$ & 0.855 & 0.545 & 0.669 & 0.7539 & 0.0437 & 0.00537 \\
\hline
\end{tabular}

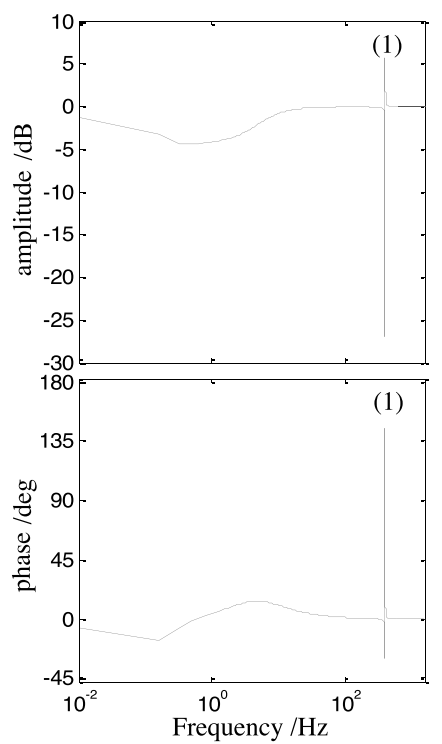

(1) PID parameters in Table .1-A

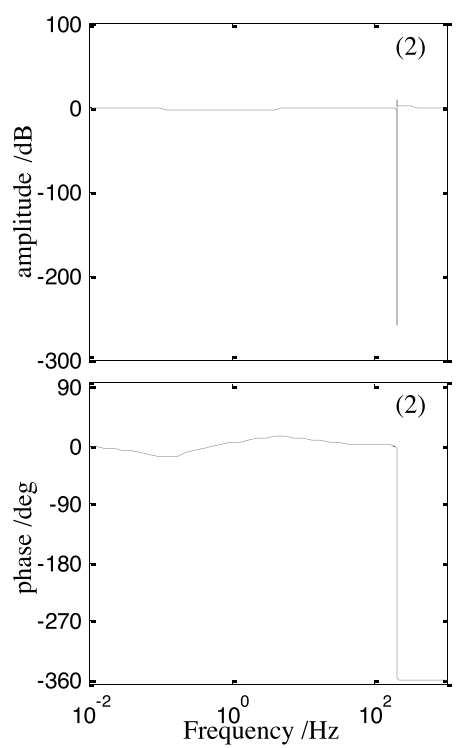

(2) PID parameters in Table .1-B

Figure 7. $C_{N}$ close loop transfer function bode diagrams contrast. 
In Figure 7-(1), the amplitude frequency characteristic curve is near $0 \mathrm{~dB}$ besides the filter frequency $400 \mathrm{~Hz}$; it shows that the system output can respond to the input well. The amplitude frequency value is far less than $-3 \mathrm{~dB}$ in $400 \mathrm{~Hz}$; it can filter well in this frequency. The phase frequency value is always above $-180^{\circ}$, and it shows that the system has good stability after filtering point. But if the PID parameters have small changes, like Table 1-B, only $T_{d}$ was changed, while the controller closed loop transfer function bode diagrams have changed remarkably in Fig. 7-(2). From the phase frequency characteristic curve, it can be seen that the phase lags one period and will make the maglev flywheel control system instable. So, suitable $\mu$ should be chosen and corresponding groups PID parameters should be selected according to the flywheel different rotating frequency to implement switch control.

Figure 8 gives the real-time switching control simulation for Figure 6 using MATLAB.

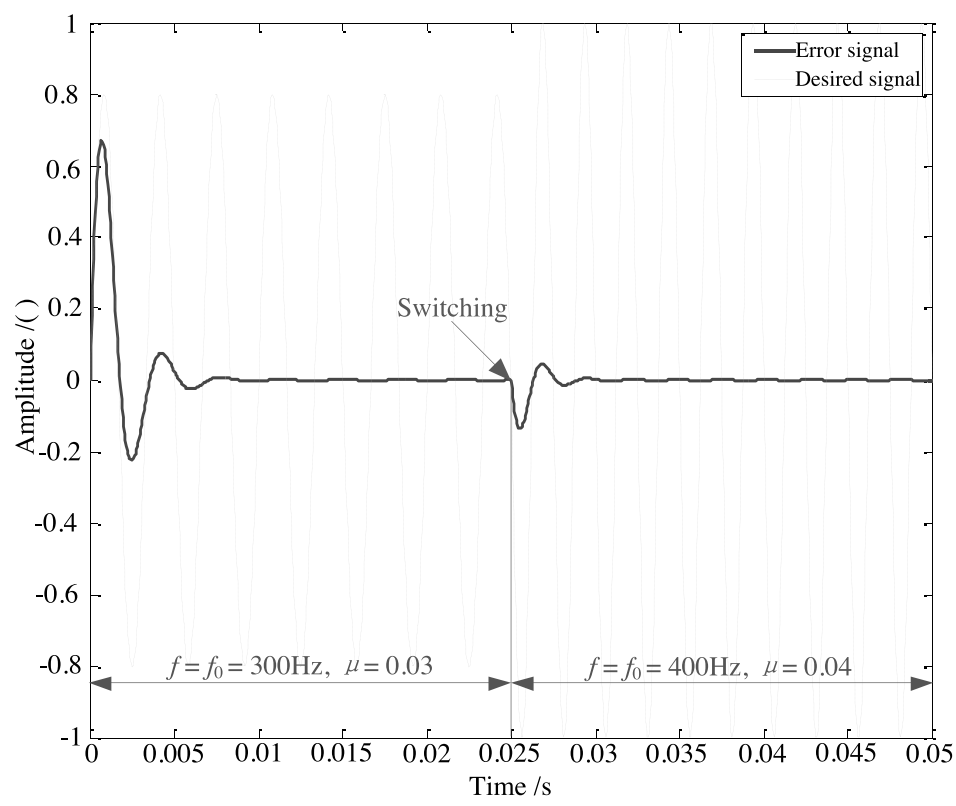

Figure 8. The real-time switching control simulation.

In Figure 8, the amplitude and the frequency of the desired signal are dynamically changed, to track the desired signal well, assuming the compensation frequencies are $300 \mathrm{~Hz}$ and $400 \mathrm{~Hz}$, and the step sizes are selected to be 0.03 and 0.04, respectively, and the PID parameters are selected in Table $1(400 \mathrm{~Hz}$ for A and $300 \mathrm{~Hz}$ for B). When the desired signal has changed and the switch compensation control is completed, the control is stable, and the error signal can convergent again quickly. 


\section{EXPERIMENT RESULT}

Now the configuration of the active compensation control system is given in Figure 9.

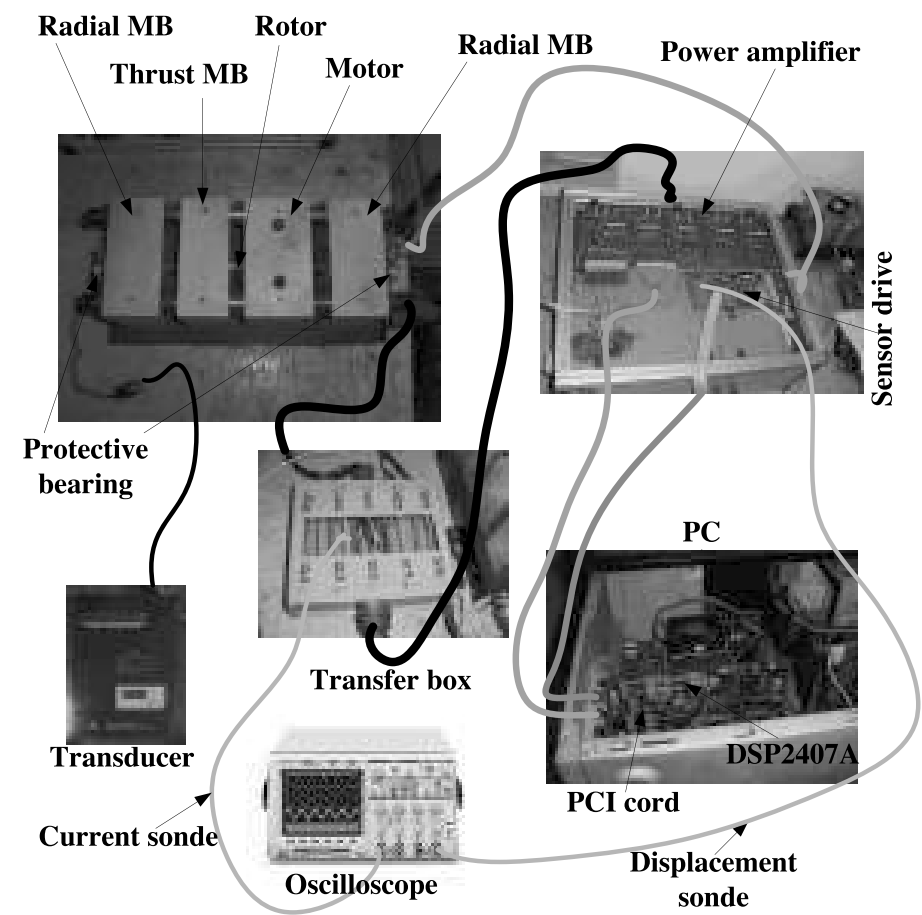

Figure 9. Real closed-loop manipulative system of AMB system.

where PC and digital control are the keys of implementing active compensation control. The action of transducer is to drive the motor rotation, and the action of transfer box is debugged and measured conveniently. Relevant algorithms are programmed in PC and DSP.

Figure 10 and Figure 11 give the displacement curves and control current curves at $300 \mathrm{~Hz}$ and $400 \mathrm{~Hz}$ with and without compensation, respectively. The step sizes and the PID parameters are consistent with the simulation parameters in Figure 9.

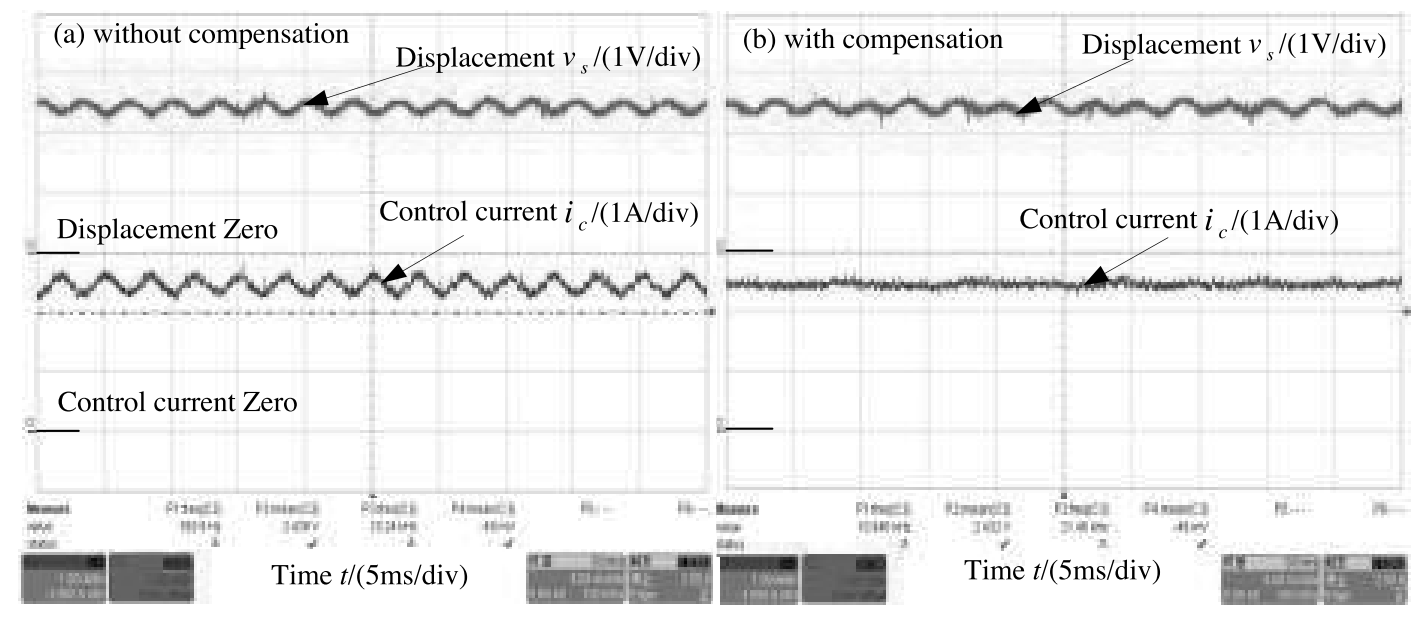

Figure 10. Displacement curves and control current curves at $300 \mathrm{~Hz}$ with and without compensation. 


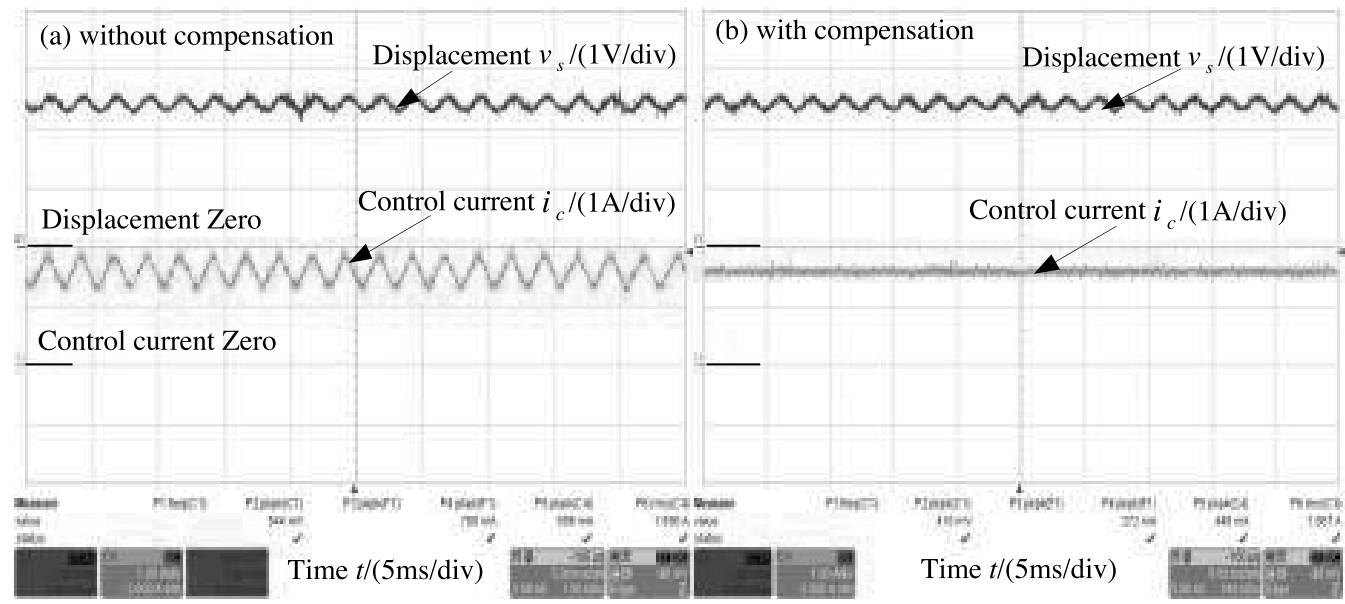

Figure 11. Displacement curves and control current curves at $400 \mathrm{~Hz}$ with and without compensation.

In Figure 10 and Figure 11, if the rotational frequency $f$ equals the compensation frequency $f_{0}$, the control currents tend to be a straight line basically, and the minimum inertia force compensation is achieved. The control current amplitude attenuates about $90 \%$ when the rotation frequency is $300 \mathrm{~Hz}$, and it attenuates about $95 \%$ when it is $400 \mathrm{~Hz}$. The control current amplitudes attenuation illustrate that the unbalance sinusoidal signals are filtered basically in the flywheel displacement, and the active control effect is reduced.

Now the vibration switch compensation for maglev flywheel system is analyzed, assuming the flywheel rotational frequency is $400 \mathrm{~Hz}$, and the switch compensation is executed in two different controllers. The two controller parameters are shown in Table 2, and the displacement curves and control current curves before and after switch compensation are given in Figure 12.

Table 2. The two controller parameters.

\begin{tabular}{cccccccc}
\hline$f_{0}$ & $\mu$ & $K p$ & $T_{i}$ & $i_{d}$ & $T_{f}$ & $T_{l}$ & $\alpha$ \\
$380 \mathrm{~Hz}$ & 0.038 & 0.625 & 0.545 & 0.729 & 0.7539 & 0.0437 & 0.00537 \\
$420 \mathrm{~Hz}$ & 0.042 & 0.665 & 0.545 & 0.629 & 0.7539 & 0.0437 & 0.00537 \\
\hline
\end{tabular}

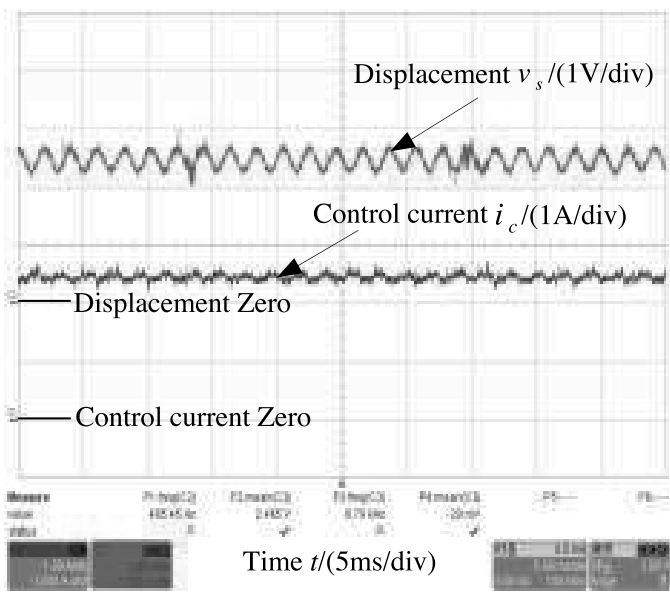

(a) compensation frequency is $380 \mathrm{~Hz}$

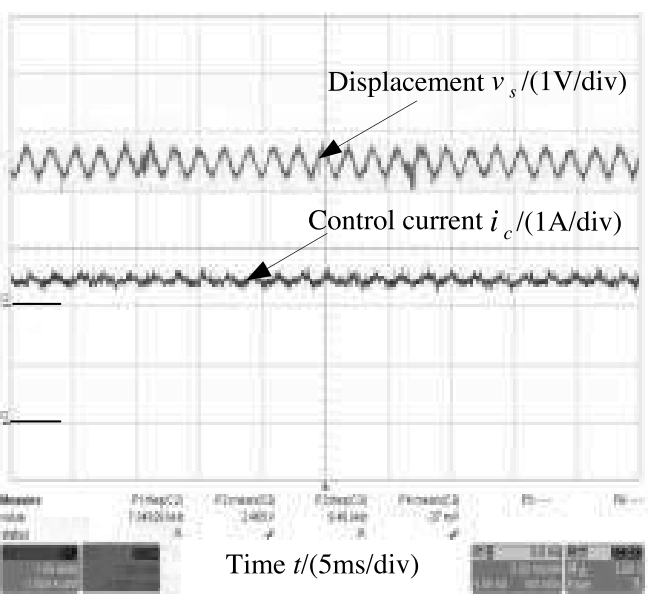

(b) compensation frequency is $420 \mathrm{~Hz}$

Figure 12. Displacement curves and control current curves before and after switch compensation. 
In Figure 12, the control current amplitudes attenuate about $80 \%$, and the results are consistent with the simulation in Figure 4, in which the filter compensation is not thorough. Besides, the switch process is stable when the rotational frequency is up to $400 \mathrm{~Hz}$.

\section{CONCLUSION}

In this paper, one vibration switching compensation controller is designed. The controller includes some having the same structure control modules, and every module is composed by the standard LMS algorithm and the incomplete derivative PID control algorithm. The switch compensation is implemented using different compensation controller according to the rotational frequency change. Simulation and experiment results verify the feasibility and effectiveness of the switch compensation strategy, can reduce the control current amplitude, and make the flywheel rotate around the inertial principal axis as much as possible.

\section{ACKNOWLEDGMENT}

This work was supported in part by the State Grid Corporation of China Science and Technology Project "Research on key technologies of intelligent diagnosis and early warning of electric vehicle charge-discharge faults and operation and maintenance service system".

\section{REFEREENCES}

Aliyu, M.; Alkassim, M. \& Salman, M. (2015). A p-norm variable step-size LMS algorithm for sparse system identification. Signal Image and Video Processing, 9: 1559-1565.

Bogdanovic, N.; Plata-Chaves, J. \& Berberidis, K. (2014). Distributed Incremental-Based LMS for Node-Specific Adaptive Parameter Estimation. IEEE Trans. Signal Process. 62: 5382-5397.

Chen, S.C.; Le, D.K. \& Nguyen, V.S. (2014). Inductive Displacement Sensors with a Notch Filter for an Active Magnetic Bearing System. Sensors 14:12640-12657.

Fang, J.C.; Xu, X.B. \& Xie J.J. (2015). Active vibration control of rotor imbalance in active magnetic bearing systems. J. Vibration and Control, 21: 684-700.

Feuer, A. \& Weinstein, E. (1985). Convergence Analysis of LMS Filters with Uncorrelated Gaussian Data. IEEE Trans. Acous, Speech, Signal Process. 33: 222-229.

Gao H. \& Song W. (2015). Analysis on unbalance vibration and compensation for maglev flywheel, International Journal of Applied Electromagnetics and Mechanics, 50: 177-187.

Ghogho, M. \& Ibnkahla, M. (1998). Analytic Behavior of the LMS Adaptive Line Enhancer for Sinusoids Corrupted by Multiplicative and Additive Noise. IEEE Trans. Signal Process. 46: 2386-2393.

He L.; Deng Z. \& Li H. (2015). The digital penalized LMS deconvolution method for TPC X-ray polarimeter signal processing. J. Instrumentation, 10: 1-8.

Liu W.; Kang, D. \& Zhang, C.N. (2016). Design of a High-T-c Superconductive Maglev Flywheel System at 100-kW Level, IEEE Trans. Applied Superconductivity, 26:1-5.

Martinek, R; Kahankova, R; Nazeran, H; Konecny, J; Jezewski, J; Janku, P; Bilik, P; Zidek, J; Nedoma, J \& Fajkus, M. (2017). Non-Invasive Fetal Monitoring: A Maternal Surface ECG Electrode Placement-Based Novel Approach for Optimization of Adaptive Filter Control Parameters Using the LMS and RLS Algorithms. Sensors. 17: 1-31.

Niemann, A.C.; van Schoor, G. \& du Rand, C.P. (2013). A Self-Sensing Active Magnetic Bearing Based on a Direct Current Measurement Approach. Sensors 13:12149-12165.

Raymond M.S.; Kasarda M.E. \& Allaire P.E. (2015). Small Signal Analysis of Power Systems With Wind and Energy Storage Units. IEEE Trans. power system. 30:298-305.

Shi, J. \& Zmood, R. (2002). The Direct Method for Adaptive Feed-forward Vibration Control of Magnetic Bearing Systems. 7th International Conf. Control, Automation, Robotics and Vision, 675-680. 
Ting, L.K.; Cowan, C. \& Woods, R.E. (2004). LMS coefficient filtering for time-varying chirped signals. IEEE Trans. Signal Process. 52: 3160-3169.

Widrow , B.; Mcool \& J. Ball, M. (1975). The Complex LMS Algorithm, Proceedings of the IEEE, 719-720.

Xiao, Y. \& Tadokoro, Y. (1995). LMS-based Notch Filter for the Estimation of Sinusoidal Signals in Noise. Signal Process. 46: $223-231$.

Xu Y. P.; Zhou J. \& Di L. (2016). Active magnetic bearings dynamic parameters identification from experimental rotor unbalance response. Mechanical Systems and Signal Processing, 83: 228-240.

Yao, D.; Wang, J.W. \& Liu, Y. (2015). Enhancing working performance of active magnetic bearings using improved fuzzy control and Kalman-LMS filter. J. intelligent \& fuzzy systems, 29: 1343-1353.

Submitted: 24/02/2017

Revised: $\quad 29 / 07 / 2018$

Accepted: 23/12/2018 


\title{
تعويض تحول الاهتزاز لبطارية ماجليف استناداً إلى خوارزمية LMS القياسية
}

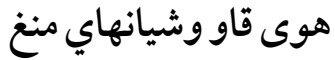 \\ جامعة نانجينغ للبريد و الاتصالات، نانجينغ، الصين
}

\section{الخلاصة}

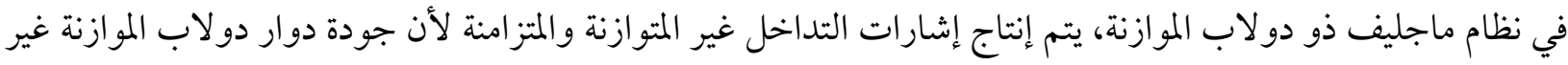

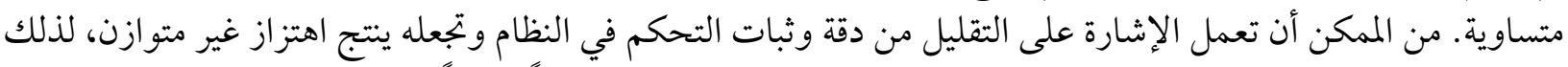

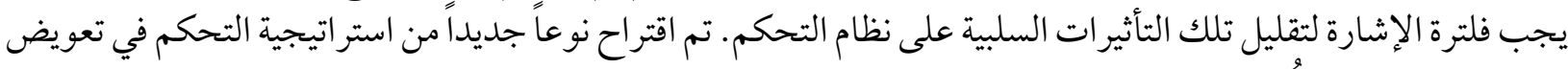

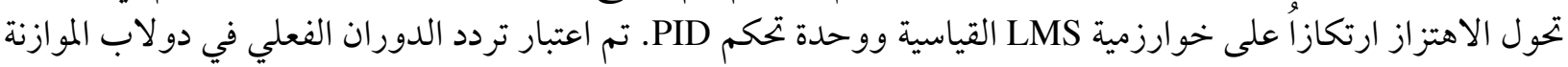

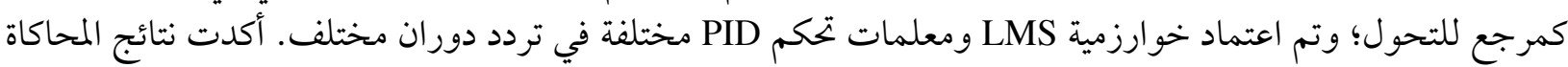

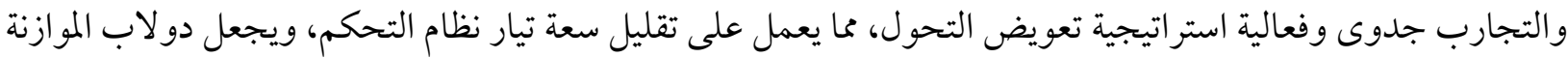
يدور حول المحور الرئيسي قدر الإمكان. 